\title{
Impact of Heavy Flavor PDFs at the LHC
}

\section{B. Clark, E. Godat, T. Ježo, K. Kovařík, A. Kusina, F. Lyonnet, ${ }^{1}$ F. I. Olness, ${ }^{1}$,} I. Schienbein, J. Y. Yu ${ }^{1 \dagger}$

${ }^{1}$ Southern Methodist University, Dallas, TX 75275, USA

${ }^{2}$ Universitá di Milano-Bicocca and INFN, Sezione di Milano-Bicocca, Piazza della Scienza 3, 20126 Milano, Italy

${ }^{3}$ Institut für Theoretische Physik, Westfälische Wilhelms-Universität Münster, Wilhelm-Klemm-Straße 9, D-48149 Münster, Germany

${ }^{4}$ Laboratoire de Physique Subatomique et de Cosmologie, Université Grenoble-Alpes, CNRS/IN2P3, 53 avenue des Martyrs, 38026 Grenoble, France

The discovery potential of the LHC depends sensitively on the input PDFs. Despite extensive efforts, the precision of the heavy quark distributions have significant uncertainties. We illustrate some of these issues for the case of the strange quark whose extraction is intertwined with the corresponding nuclear corrections. The use of LHC W/Z production data from both proton and heavy-ion beams could substantively improve the precision of both the PDFs and the nuclear corrections.

XXIV International Workshop on Deep-Inelastic Scattering and Related Subjects

11-15 April, 2016

DESY Hamburg, Germany

\footnotetext{
* Speaker.

${ }^{\dagger}$ This work was supported by the U.S. Department of Energy under Grant No. DE-SC0010129, and by Projet international de cooperation scientifique PICS05854 between France and the U.S.
} 

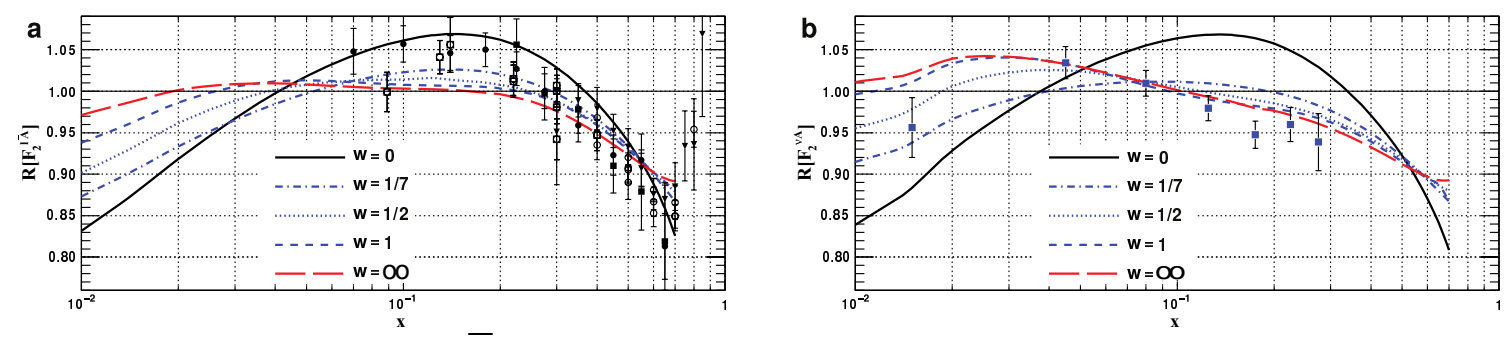

Figure 1: Predictions from Ref. [1] for the nuclear correction factors $(A=F e)$ with (a) $R\left[F_{2}^{\ell A}\right] \simeq$ $F_{2}^{\ell A} / F_{2}^{\ell N}$ and (b) $R\left[F_{2}^{v A}\right] \simeq F_{2}^{V A} / F_{2}^{V N}$ as a function of $x$ for $Q^{2}=5 \mathrm{GeV}^{2}$. The data points displayed in (a) are from BCDMS and SLAC experiments and those displayed in (b) come from the NuTeV experiment.

\section{Introduction}

The 2015 run of the Large Hadron Collider (LHC) at $\sqrt{s}$ of $13 \mathrm{TeV}$ opened up a new frontier for discovery. As the LHC experiments accumulate rising statistics, it is essential for theorists to match these efforts with increasingly precise theoretical predictions. Essential elements necessary for such predictions are accurate Parton Distribution Functions (PDFs). Despite decades of effort, there are still PDF components which can benefit from improvements. In particular, the strange content of the proton has relatively large uncertainties, yet this plays an increasingly important role in the LHC physics. In this short note we will focus on the strange PDF flavor, look at its extraction, and consider how we might improve its determination.

\section{Flavor Differentiation}

The challenge in extracting the strange quark PDF is to distinguish it underneath the dominant up and down components. One process which is especially sensitive to the strange PDF is the neutrino-nucleon DIS process with a pair of opposite-sign muons in the final state: $v N \rightarrow X \mu^{+} \mu^{-}$. This process is sensitive to the underlying partonic process $W^{+} s \rightarrow c$ (with the charm decaying leptonically), and using sign-selected neutrino beams the strange and anti-strange PDF can be separately determined. As the neutrino cross sections are typically small, these experiments are performed on heavy nuclear targets such as iron or lead; thus, the extraction of the strange quark PDF is intertwined with the corresponding nuclear corrections.

In fact, these nuclear corrections are not well determined. In Fig. 1 we display the nuclear correction factors for charged lepton DIS data (left) and neutrino DIS data (right) on iron. If we take these results at face value, they appear to be incompatible.[1] The curves in Fig. 1 show a parameterization of the nuclear correction factor which attempts to interpolate between the charged lepton DIS data $(w=0)$ and neutrino DIS data $(w=\infty)$. In Fig. 1-a we show the data points from the charged lepton DIS experiments (BCDMS and SLAC), and in Fig. 1-b we show the neutrino DIS data $(\mathrm{NuTeV})$. Different nuclear corrections will result in different extracted PDFs, and thus yield correspondingly different theoretical predictions. 


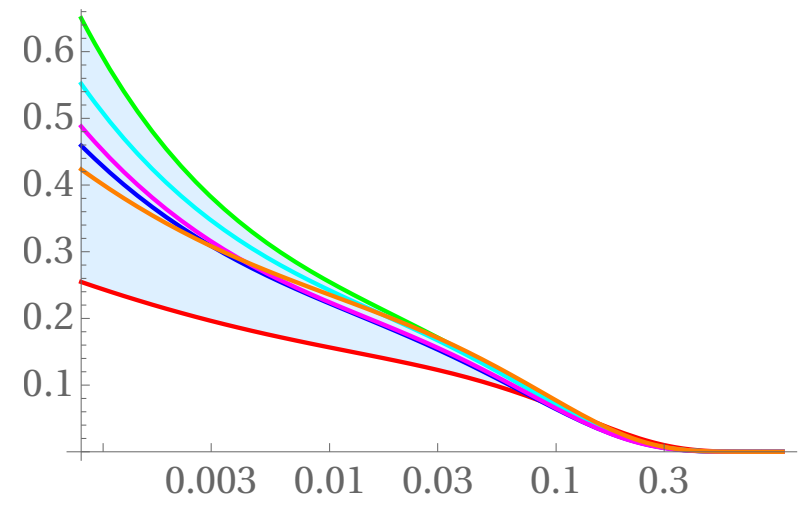

(a) The strange PDF at $Q=3 \mathrm{GeV}$ vs. $x$ for the set of PDFs displayed in Fig. 1. The solid lines show the central values, and the span of the curves provides a measure of the nuclear PDF uncertainty.

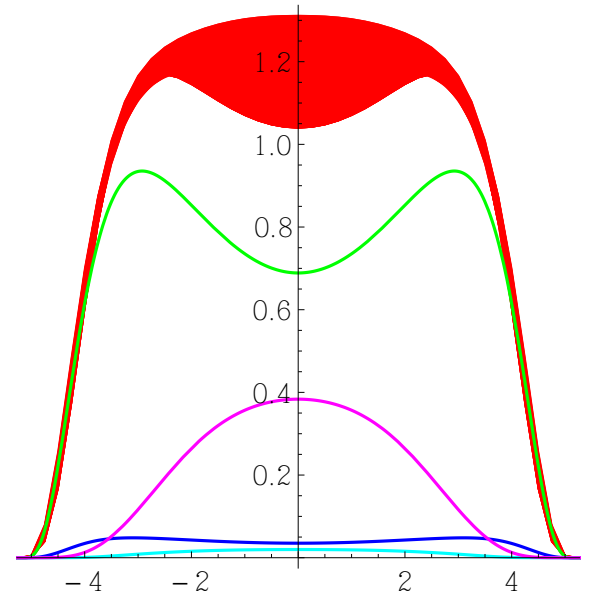

(b) The scaled cross section $d \sigma / d y$ for $W^{+}$ production at the $\mathrm{LHC}(\sqrt{s}=13 \mathrm{TeV})$. The upper (red) curve is the total with uncertainties. and the lower curves are (in order) the $u \bar{d}$ (green), $c \bar{s}$ (magenta), $u \bar{s}$ (blue), $c \bar{d}$ (cyan) contributions.

Figure 2:

\section{Impact on LHC Processes}

We now take the collection of PDFs from Fig. 1 to assess the uncertainty of the strange quark PDF and its impact on LHC processes. In Fig. 2-a we display the strange quark vs. the momentum fraction $x$ at $Q=3 \mathrm{GeV}$; this allows us to approximately gauge the relative uncertainty which can be as large as 50\% at small $x$ values. To see the effect on an LHC process, in Fig. 2-b we show the total cross section $d \sigma / d y$ for $W^{+}$production as well as the individual partonic contributions $\{u \bar{d}, c \bar{s}, u \bar{s}, c \bar{d}\}$ using a simple leading order assessment (see Refs. [8-10] for details). In this preliminary analysis, the uncertainty band (red) shown in the plot is composed of the collection of PDFs from Fig. 1. Due to the high energy at the LHC (as compared to the Tevatron), the heavier quarks play a significant role and can contribute $\sim 30 \%$ or more. Thus, a large uncertainty in the strange quark PDF will propagate into the uncertainty of $d \sigma / d y$. As W/Z production is one of the "standard candles" at the LHC, this simple exercise underscores the importance of improving strange quark PDF determination to facilitate incisive measurements which form the foundations for new physics searches.

\section{4. nCTEQ Parton Distribution Functions}

As the LHC is producing heavy ion collisions with proton-lead and lead-lead, we can approach this issue from an alternate direction. If we can refine the measurement of the nuclear correction factors, this can then improve the extraction of the strange PDF. The nCTEQ PDF project has taken the proton PDF global analysis as a starting point and extended it in the nuclear dimension. Specifically, at scale $\mu=Q_{0}$ we generalize the initial proton PDFs $f_{p}\left(x, Q_{0}\right)$ to include the nuclear 

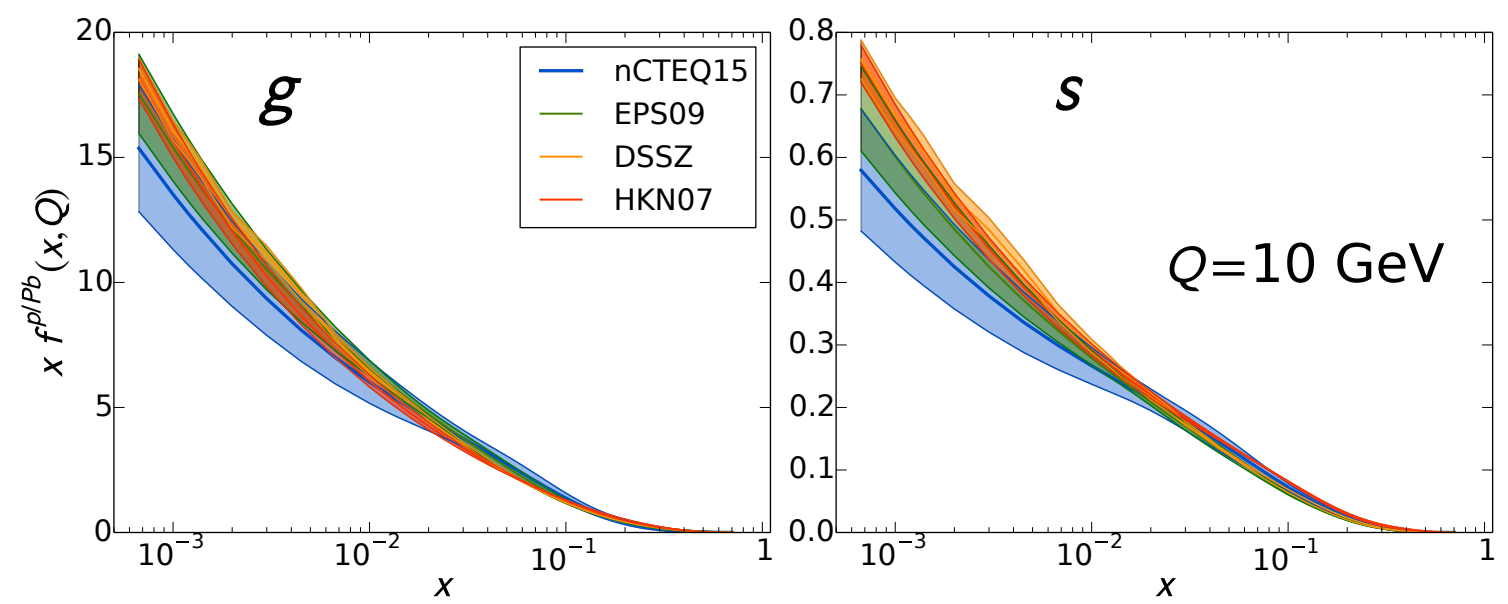

Figure 3: Selected nCTEQ15 PDFs (gluon, strange) vs. $x$ at $Q=10 \mathrm{GeV}$ for a proton in a lead nucleus, $f^{p / P b}(x, Q)$. We display the central values (solid line) and the uncertainty band (shaded region) for nCTEQ15[2], HKN07[3], EPS09[4] and DSSZ[5].

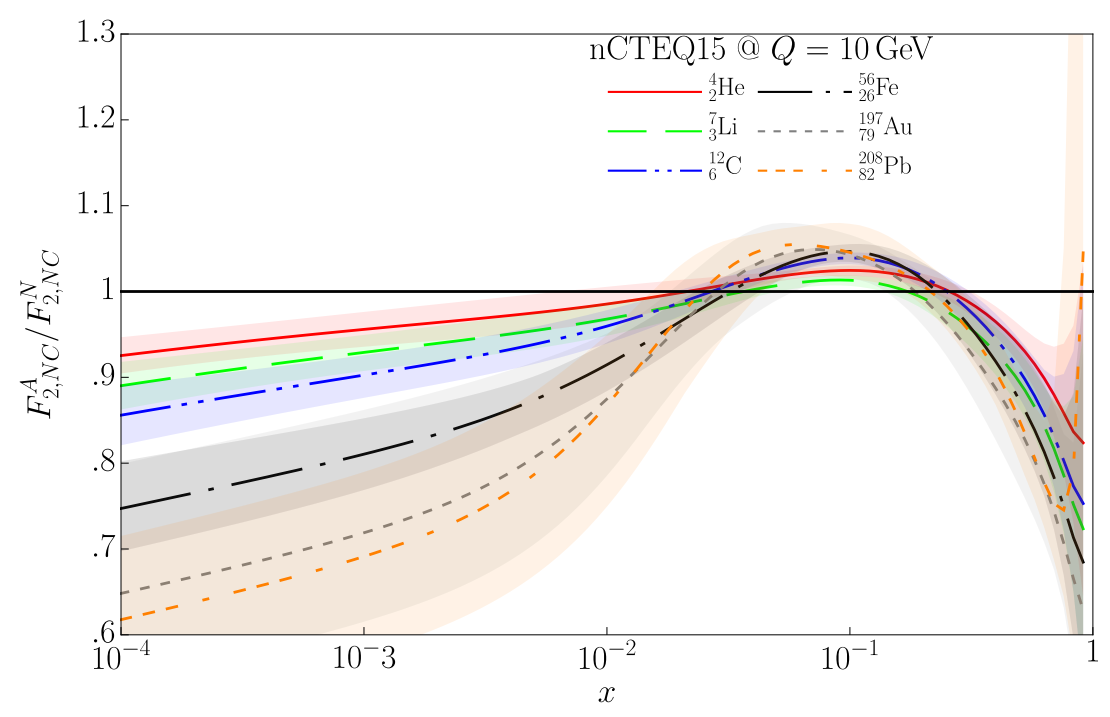

Figure 4: The nuclear correction factors $F_{2}^{A} / F_{2}^{N}$ for the nCTEQ15[2] PDFs with uncertainty bands.

$A$-dependence $f_{N}\left(x, Q_{0}, A\right)$, then evolve these with the DGLAP evolution equations, and fit a variety of nuclear targets. The most recent result of this analysis is the nCTEQ15 PDF set which includes error PDF sets generated using the Hessian eigenvector method.[2] This allows us to quantitatively assess the uncertainty due to the nuclear corrections. ${ }^{1}$

For example, in Fig. 3 we show selected PDF flavors for a proton in a lead nucleus vs. $x$ at a scale $Q=10 \mathrm{GeV}$; both the central value (solid line) and the uncertainty bands (shaded regions) are show. ${ }^{2}$ For comparison, we also display the results from other recent nPDF determinations including HKN07, EPS09, and DSSZ. We observe the four bands are in very good agreement

\footnotetext{
${ }^{1}$ In principle this can be convoluted with the proton PDF uncertainties; however, since the nuclear uncertainties typically dominate we find in practice the total is close to the nuclear uncertainty.

${ }^{2}$ Note, this analysis includes only the $\ell^{ \pm} N$ DIS data due to the issues highlighted in Ref. [1].
} 


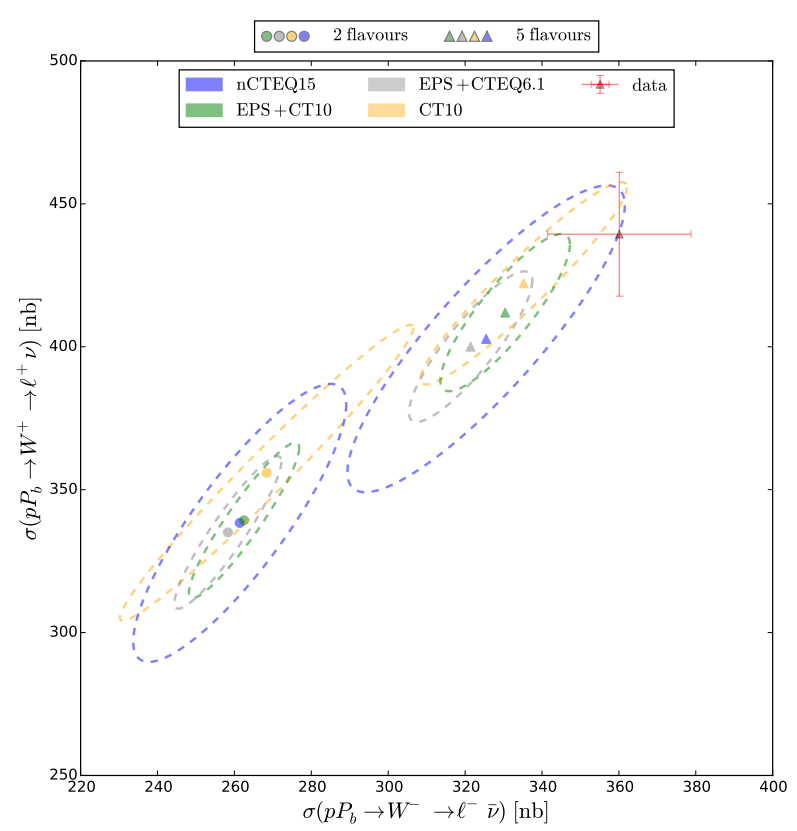

Figure 5: Preliminary results for the comparison of $W^{ \pm}$cross section correlations for only one family $(u, d)$ of quarks (circles) and all families (triangles) in proton-lead collisions.[11]

at larger $x$ values where there are strong constraints from data, but the bands begin to diverge at smaller $x$ values where the constraints are reduced. For $W^{ \pm}$production at the LHC the characteristic $x$ values are $\sim 10^{-3}$ to $\sim 10^{-2}$ depending on the energy, and Fig. 3-b shows quite a bit of spread in this $x$ region for the strange PDF.

Using the nCTEQ15 PDF sets we can construct the uncertainty for any physical observable as a function of $A$. In Fig. 4 we display the ratio $R=F_{2, N C}^{A} / F_{2, N C}^{N}$ for a selection of nuclear targets as a function of $x$. The $x$ regions are typically divided into a Fermi region $(R>1$ at very high $x)$, an EMC region $(R<1$ at large $x)$, an anti-shadowing region $(R>1$ at intermediate $x)$, and a shadowing region $(R<1$ at small $x)$. As we move from light nuclei $(\mathrm{He})$ to heavier nuclei $(\mathrm{Pb})$ we observe the magnitude of the nuclear correction is uniformly increasing. If we can make use of different nuclear measurements to reduce the uncertainty on the $R$ correction factor, this can help improve the extraction of the PDFs from the nuclear targets, which in turn will improve the determination of all the PDF flavors, especially the strange quark.

\section{W/Z Boson Production at the LHC}

The production of W/Z bosons at the LHC in both the proton and heavy-ion channels provides a unique opportunity to untangle the nuclear corrections from the strange PDF. In Fig. 5 we display the (preliminary) correlations of the $W^{ \pm}$cross section for proton-lead collisions using various PDF sets. $[6,7,11]$ Specifically, we display the central value and error ellipses of nCTEQ15, the EPS09 
corrections with CT10 PDFs, the EPS09 corrections with CTEQ6.1 PDFs, ${ }^{3}$ and the CT10 PDFs without any nuclear correction. While there is some spread in these central values, the results fall well within the error ellipses.

To emphasize the role of the heavier quark contribution $\{s, c, b\}$, we also display the results using only the first generation $\{u, d\}$ quarks (circles), along with the 5-flavor result (triangles). The difference between these two results is striking, and dominantly due to the strange quark. This observation demonstrates that the LHC $W$ production process is sensitive to the heavier quark PDFs (including the strange quark) at a level beyond the nuclear uncertainties.

Therefore, by combining LHC W/Z data from both proton and heavy ion beams together with the existing measurements (such as those used in the nCTEQ15 analysis), we can make significant strides in resolving the outstanding issues regarding the nuclear corrections and the strange quark PDF. This is a promising avenue of investigation to pursue, and a more detailed study is currently being completed.[11]

\section{References}

[1] K. Kovarik, I. Schienbein, F. I. Olness, J. Y. Yu, C. Keppel, J. G. Morfin, J. F. Owens and T. Stavreva, "Nuclear corrections in neutrino-nucleus DIS and their compatibility with global NPDF analyses," Phys. Rev. Lett. 106, 122301 (2011) [arXiv:1012.0286 [hep-ph]].

[2] K. Kovarik et al., "nCTEQ15 - Global analysis of nuclear parton distributions with uncertainties in the CTEQ framework,” Phys. Rev. D 93, no. 8, 085037 (2016) [arXiv:1509.00792 [hep-ph]].

[3] M. Hirai, S. Kumano \& T.-H. Nagai, "Determination of nuclear parton distribution functions and their uncertainties in NLO,” Phys. Rev. C 76, 065207 (2007) [arXiv:0709.3038 [hep-ph]].

[4] K. J. Eskola, H. Paukkunen and C. A. Salgado, "EPS09: A New Generation of NLO and LO Nuclear Parton Distribution Functions,” JHEP 0904, 065 (2009) [arXiv:0902.4154 [hep-ph]].

[5] D. de Florian, R. Sassot, P. Zurita and M. Stratmann, "Global Analysis of Nuclear Parton Distributions," Phys. Rev. D 85, 074028 (2012) [arXiv:1112.6324 [hep-ph]].

[6] R. Gavin, Y. Li, F. Petriello and S. Quackenbush, "FEWZ 2.0: A code for hadronic Z production at NNLO," Comput. Phys. Commun. 182, 2388 (2011) [arXiv:1011.3540 [hep-ph]].

[7] R. Gavin, Y. Li, F. Petriello and S. Quackenbush, "W Physics at the LHC with FEWZ 2.1," Comput. Phys. Commun. 184, 208 (2013) [arXiv:1201.5896 [hep-ph]].

[8] A. Kusina, F. I. Olness, I. Schienbein, T. Jezo, K. Kovarik, T. Stavreva and J. Y. Yu, "Hybrid scheme for heavy flavors," Phys. Rev. D 88, no. 7, 074032 (2013) [arXiv:1306.6553 [hep-ph]].

[9] A. Kusina et al., "Strange Quark PDFs and Implications for Drell-Yan Boson Production at the LHC," Phys. Rev. D 85, 094028 (2012) doi:10.1103/PhysRevD.85.094028 [arXiv:1203.1290 [hep-ph]].

[10] F. Olness and I. Schienbein, "Heavy Quarks: Lessons Learned from HERA and Tevatron,” Nucl. Phys. Proc. Suppl. 191, 44 (2009) [arXiv:0812.3371 [hep-ph]].

[11] A. Kusina, et al., "Vector boson production in $\mathrm{pPb}$ and $\mathrm{PbPb}$ collisions at the LHC and its impact on nCTEQ15 PDFs," SMU-HEP-16-12, In preparation.

\footnotetext{
${ }^{3}$ We display the CTEQ6.1 PDFs as these are the baseline for the EPS09 analysis.
} 